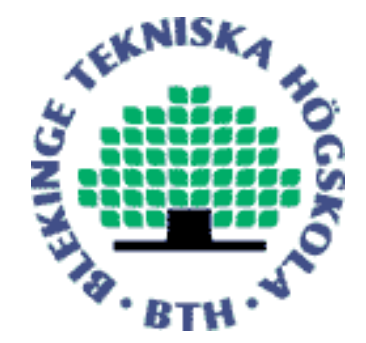

Copyright (C) 2010 IEEE.

Citation for the published paper:

A Middleware Framework for Communication in Cognitive Radio Networks

Alexandru Popescu, David Erman, Markus Fiedler, Adrian Popescu, Demetres Kouvatsos

International Congress On Ultra Modern Telecommunications and Control Systems

2010 Moscow

This material is posted here with permission of the IEEE. Such permission of the IEEE does not in any way imply IEEE endorsement of any of BTH's products or services Internal or personal use of this material is permitted. However, permission to reprint/republish this material for advertising or promotional purposes or for creating new collective works for resale or redistribution must be obtained from the IEEE by sending a blank email message to pubs-permissions@iee.org.

By choosing to view this document, you agree to all provisions of the copyright laws protecting it. 


\title{
A Middleware Framework for Communication in Cognitive Radio Networks
}

\author{
Alexandru Popescu ${ }^{\dagger \ddagger}$, David Erman ${ }^{\dagger}$, Markus Fiedler $^{\dagger}$, Adrian Popescu ${ }^{\dagger}$ and Demetres Kouvatsos ${ }^{\ddagger}$ \\ $\dagger$ Dept. of Communications and Computer Systems \\ School of Computing \\ Blekinge Institute of Technology \\ 37179 Karlskrona, Sweden \\ $\ddagger$ Dept. of Computing \\ School of Informatics \\ University of Bradford \\ Bradford, West Yorkshire BD7 1DP, United Kingdom
}

\begin{abstract}
-
Cognitive radio networks are expected to address and resolve important operational challenges such as opportunistic spectrum access, spectrum and network heterogeneities and requests for the provision of diverse Quality-of-Service (QoS) for different applications. Accordingly, a number of network functionalities are required to take into account these challenges towards the efficient management of cognitive radio networks. This paper advances a new architecture for the management of cognitive radio networks. The architecture is designed and developed at the application layer and incorporates sensing and prediction, addressing and routing, middleware and decision making.

Accordingly, a number of management functions are required to address the challenges associated with the management of cognitive radio networks. The paper provides an analysis of these components as well.
\end{abstract}

\section{INTRODUCTION}

Cognitive Radio Networks (CRNs) are emerging as a viable solution to solve spectrum shortage problems. By configuring different transmission parameters (e.g., frequency band, waveform, transmit power) on the fly, wireless networks resources can be shared with licensed users in an opportunistic manner. Temporarily unused portions available in an $n$-dimensional space can be identified and used for communication and consequently exploiting under-utilized spectrum portions (socalled "spectrum holes"). Examples of such dimensions are frequency, power, time, geographical space, code and angle [1]-[3].

A Cognitive Radio Device (CRD) has particular facilities in terms of hardware, software and signal processing, able to adapt and to learn from experience. The device collects information about the surrounding environment, sensing and understanding it as well as to actively change the mode of operation to adapt to the particular environmental conditions. The mode of operation refers to characteristics like, e.g., frequency, modulation and transmission power. Furthermore, the CRD is requested to be aware of the rules within which it can operate fulfilling the needs and privileges of diverse operators and users.

Given the extreme complexity present in the process of CRN management we suggest a new solution for communication

978-1-4244-7286-4/10/\$26.00 (C) 2010 IEEE in CRNs, which is based on the cognitive framework for the control of CRNs described in [4]. The cognitive framework is a software framework, used to map the high-level requirements to the underlaying network by using the cognitive process. There are three layers in this framework, which are called "End-to-end Goals", "Cognitive Process" and "Software Adaptive Network". The highest layer handles the specification of e2e goals, which are mapped to diverse requirements or policies for the cognition layer. The cognition layer uses sensors to monitor the network. This is further controlling the software adaptive network. It is important to mention that a cognitive process can operate in a centralized or distributed way, each of them with specific advantages and drawbacks.

By using several dimensions in the process of identification, management and routing the paper advances a new architecture for the management of cognitive radio networks that is implemented at the application layer. Operations like spectrum sensing, addressing, decision making, routing and handover can be done in an $n$-dimensional space and used for knowledge representation and management of CRNs dimensions, i.e., frequency, power, geographical space, code and time.

Our solution is conceived to be used in crowded areas to maximize spectrum utilization and thus service availability, but also in disaster areas or military operations where no infrastructure is available. A further vision for this architecture is to be part of future generations cellular phones and networks. Through implementation at every base station and mobile device, a huge increase in resource utilization can be achieved. This would allow users and operators to efficiently utilize all opportunities in the available spectrum and not solely the operator allotted bands, hence reducing the probability for blockage in densely populated cells. The research challenges are on sensing and prediction, addressing and routing, decision making and middleware.

The rest of the paper is organized as follows. Section 2 introduces the suggested architectural solution together with the main elements involved. The MD-CAN system and the associated addressing system used in our architecture is described in section 3. Section 4 develops on the cognitive engine together with a pseudocode overview of the operational algorithms. 
Section 5 provides the general concept of cognitive routing. In section 6 the main elements of the multi-dimensional routing and optimization suggested for our solution are described. Finally, section 7 concludes the paper.

\section{New Architectural APPROACH FOR COMMUNICATION IN CRNS}

There are four fundamental operations needed for the management of a cognitive radio network. These are: Spectrum Sensing, Spectrum Modeling, Spectrum Sharing and Spectrum Mobility [1], [2], [5]. Basically, the management solution used in our system is an intelligent wireless communication system, defined to represent a set of algorithms for the management of CRN operations like:

- Possibility for CRNs to select the transmission spectrum, conditioned on protecting the transmission of licensed users.

- Need for topology control, as CRNs generally lack centralized support and the available topology information is rather limited.

- Multi-dimensional transmission and handover, specifically the e2e route may consist of multiple hops in different channels placed in the $\mathrm{n}$-dimensional space considered for the management of CRNs.

The goal is to perform communication by sensing, learning, optimization and adaptation. Our architecture uses a specific middleware, a set of overlay entities and multi-dimensional routing. In other words, the complexity of communication in a CRN is disseminated to different overlays. Furthermore, a centralized entity called Support Node (SN) is introduced, providing additional functionality.

\section{A. Network Model}

The cognitive network is partitioned in a number of socalled Cognitive Radio Networks (CRNs) as shown in figure 1. Every CRN is served by a SN and contains a number of CRDs. The SN provides the basic support for the cognitive radio communication of associated CRDs. The following functions are provided by a $\mathrm{SN}$ :

- Collection of information regarding the specific CRN.

- Provision of e2e routing decision to the requesting CRDs for static routing purposes.

- Provision of bootstrapping support for newly joining unlicensed CR users.

- Provision of intra and inter-routing support among CRDs present in different CRNs.

Thus far the network model is focused on communication within a single CRN only. Communication among CRDs belonging to different CRNs is an important topic of future research. This is because of technological limits given by the spectrum sensing at devices far away, but also due to the need for exchanging large amounts of information among SNs.

The following functions are provided by a CRD:

- Spectrum sensing and collection of information regarding the particular CRN.

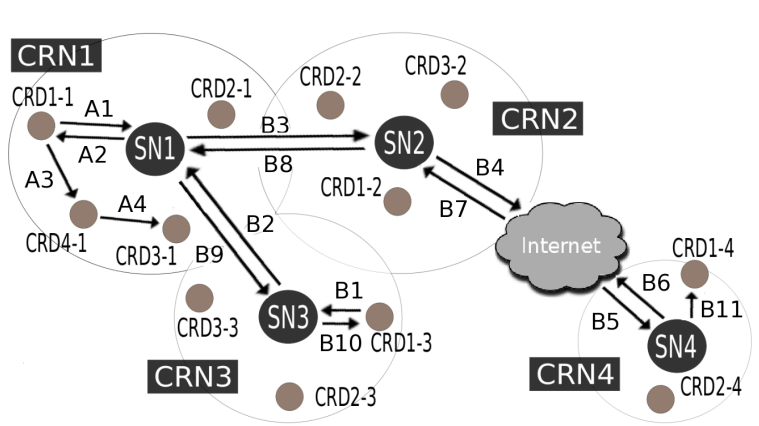

Fig. 1. Network model.

- Provision of e2e routing for a particular communication, which is based on the e2e routing decision received from the particular SN.

- Provision of e2e routing decision for dynamic routing purposes (in case the SN compiled e2e path has failed).

- Provision of support for crossing e2e routes, which includes adaptation facilities, i.e., opportunistic routing.

\section{B. Basic Scenario}

Our architecture is used to control the four fundamental operations in the management of a cognitive radio network, i.e., spectrum sensing, spectrum decision, spectrum sharing and spectrum mobility. The architecture can also control other fundamental operations associated with a CRD like, e.g., in the case of space mobility. This means that ad-hoc algorithms can be, e.g., combined with spectrum mobility algorithms to provide an e2e solution for communication. Collaboration between the CRD and $\mathrm{SN}$ is therefore vital and provides us with all necessary operations for communicating in CRNs.

A typical example scenario of communication between a $\mathrm{CRD}$ and a $\mathrm{SN}$ is shown in figure 2. A SN is responsible to populate the available free spaces in a knowledge database for a selected maximum geographical radius [6]. A CRD wishing to join a specific CRN first needs to contact the responsible $\mathrm{SN}$. Joining of unlicensed users is done through a scheduler in order to fairly partition the available spectrum holes, given that these are a limited resource. For bootstrapping purposes a CRD sends a join message to the particular SN requesting an operational zone (free space). After checking its locally stored MD-CAN entity for an available operational zone, the SN responds with all necessary data to allow the CRD to join the particular CRN. A response contains the following data: CAN virtual coordinates, channel assignments, maximum power output, list of neighboring CRDs and the ID of the CRD itself. If no operational zones are available, the joining of the CRD is rejected. A CRD is furthermore required to contact the $\mathrm{SN}$ in case adaptations of its operational parameters are necessary. This can, e.g., happen if an unexpected interferer suddenly appears, forcing the CRD to adapt in order to retain service. The adaptation data received from the $\mathrm{SN}$ is similar to the bootstrapping data, comprising similar parameters. 
In general, the primary operation of a CRD is to sense its operational environment and be aware of arising conflicts. The collected sensing and control data is propagated to the SN to update the MD-CAN overlay with current changes. Having upto-date information also improves the decision making process by compiling statistics for a group of users over a long period of time, i.e., learning from experience.

A CRD may ask to communicate with another CRD by requesting a communication route (i.e., an e2e path) from the SN. The request message sent by the CRD contains its own unique universal identification (UUID) together with the destination UUID. After checking its CRN member table the $\mathrm{SN}$ responds with a message containing the CAN cartesian coordinates of the destination CRD together with a compiled e2e route to reach it. The e $2 \mathrm{e}$ route comprises all necessary data to reach the destination, i.e., intermediate CRDs with per hop channel and power output assignments optimized according to user preferences (cost, throughput, delay). If the destination is not found in the local CRN, the responsible SN can contact other support nodes to search for the destination. However, in case this fails the request will be rejected. If the SN computed e2e path becomes invalid during an ongoing communication, a CRD can make own local decisions and adaptations on-the-fly to still be able to forward packets to the destination. These are made according to user preferences and current environmental constraints.

Figure 2 together with Algorithms 1-3 (section 5) provide an overview of the needed procedures and operation flow for the scenario described above.

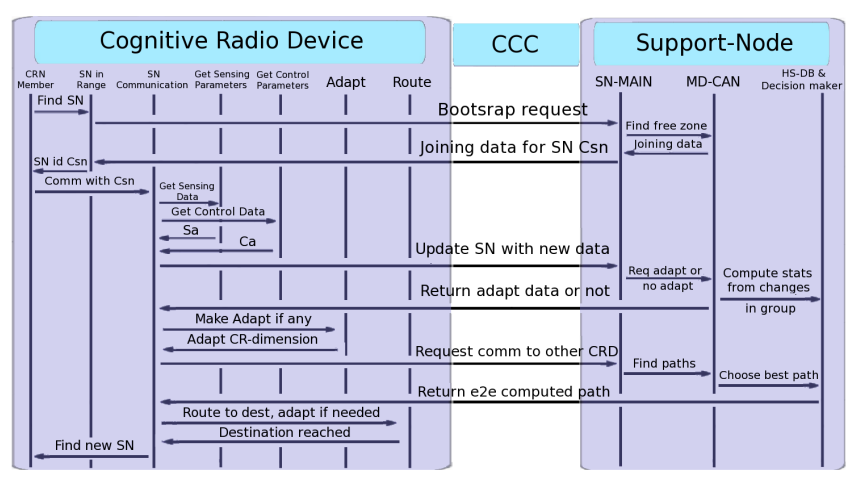

Fig. 2. Operation flow scenario.

Naturally, the diversity of network configurations and large variations in the frequency bands makes finding an appropriate e2e path between two CR users extremely challenging [7]. To achieve this, we have to consider three $\mathrm{CRN}$ routing categories [1], [2], [7]: static routing with variations in the order of hours or days, dynamic routing with temporal framings in the order of hours down to minutes and opportunistic routing (or highly dynamic) with very high variations in the order of minutes down to seconds. In short, we need to use a combined routing solution where the Decision Maker overlay uses a static routing model to locally compile the e2e path. However, if the e2e path becomes invalid local decisions have to be made by intermediate CRDs (along the path to the destination), considering dynamic or opportunistic temporal framings.

\section{Cognitive Radio Device}

The CRD architecture has the following components: middleware, software defined radio and overlays figure 3. Short description of these is as follows.

1) Middleware: The specific middleware presented in figures 3 and 4 was originally developed for seamless handover purposes. This is a software that bridges and abstracts underlying components of similar functionality, exposing it through a common API [8]. The convergence of different technologies is simplified by using an architecture based on middleware with various overlays and underlays. This offers the advantage of flexibility in present and future development of new services and applications. The main goal was to develop a testbed to facilitate the development, testing, evaluation and performance analysis of different solutions for communicating in CRNs, while requiring minimal changes to the applications using the platform. In other words, we have a software system with two sets of APIs, one for application writers and another one for interfacing various overlay and underlay systems. We can for instance develop applications that use combinations of arbitrary overlays by having, i.e., one overlay exporting an API that another overlay can use [8].

2) Software Defined Radio: The Software Defined Radio (SDR) is a device that provides most of the Radio Frequency (RF) and Intermediate Frequency (IF) functionality, also including waveform synthesis into the digital domain [9]. The ability to rapidly adapt and facilitate fast changeover between multiple SDR physical layers is provided through integrated physical and network layer capabilities. This enables a CRD to communicate with other CRDs and SNs.

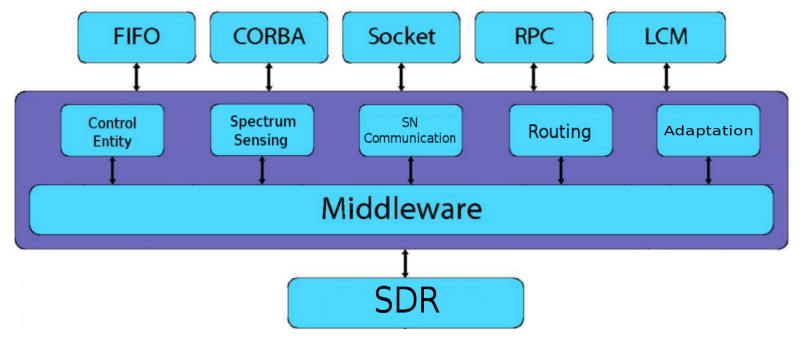

Fig. 3. Cognitive Radio Device.

3) Overlays: The following overlays are used in the CRD architecture: Control Entity, Spectrum Sensing, SNCommunication Entity, Routing and Adaptation.

1) Control entity: this overlay handles the actions related to user control, context-aware and security facilities. These actions basically refer to informing the $\mathrm{SN}$ about user context and service defined preferences as well as other relevant information for the e2e route computation. In short, this overlay offers the end user the possibility to take best decisions with the help of generic models, distributed measurements and data exchange. 
2) Spectrum Sensing: in collaboration with the SDR underlay, this overlay handles the actions related to multiband operation and fast frequency scanning, gathering information about spectrum usage. This information enables forecasting for licensed and unlicensed users as well as for the user itself (e.g., user activity, movement prediction). This is a multi-dimensional process as well. The task demands for solving a number of difficult research questions like, e.g., hidden primary user problem, detecting spread spectrum primary users, sensing duration and frequency, cooperative sensing, security.

3) SN-Communication: all communication between the terminal itself and the support node is handled by this overlay. The communication is done over a CommonControl-Channel (CCC) and through specific message types conceptualized to handle all necessary data exchange, e.g., bootstrap message, adaptation message, update message and CRD communicate message. This overlay is thus responsible for collecting all required information from the necessary overlays and properly formatting the $\mathrm{SN}$ communication messages before they are sent out on the CCC.

4) Routing: given the precompiled e2e path received from the $\mathrm{SN}$, the routing overlay is responsible for performing the actual physical routing to reach the destination. By employing suitable multi-objective, multiconstrained optimization algorithms the received e2e path is computed for ad-hoc packet routing with aggregate throughput. The destination can thus be reached according to specific QoS framings that depend on the particular domain and networking conditions. However, own decisions might be taken by this overlay, adapting to unforseen network changes in the case the precompiled e2e path is no longer valid.

5) Adaptation: while sensing the operational environment a CRD might discover that adaptations are needed in one or more $\mathrm{CR}$ dimensions in order to maintain service continuity. Consequently, a request for the necessary adaptation parameters is sent to the $\mathrm{SN}$. By interpreting the received response from the $\mathrm{SN}$ this particular overlay is then responsible for executing all changes to the affected CR-dimensions, e.g., frequency or power, in collaboration with the SDR underlay.

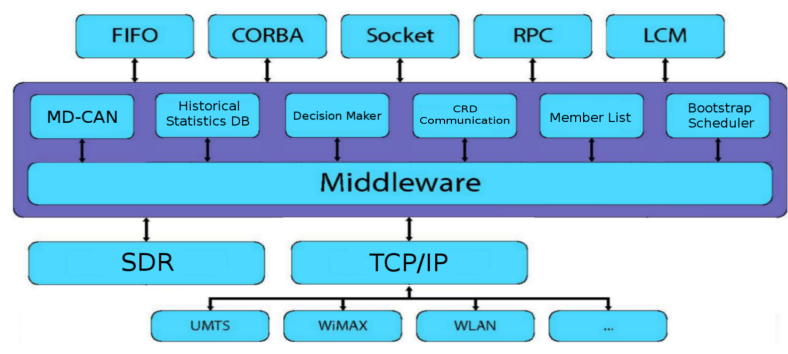

Fig. 4. Support Node.

\section{Support Node}

The Support Node (SN) (figure 4) is a centralized entity providing the following functionality:

1) Multi-Dimensional $C A N$ : this is a knowledge database for data representation of the particular multihop cognitive radio network. By changing and adapting parts of a MD-CAN, which is a multi-dimensional extension of a standard CAN implementation, we have a flexible solution to suit our particular needs for addressing and communicating in CRNs. Available operational zones (free spaces) in different CR dimensions like, e.g., frequency, power, space and code are identified and represented, thus creating service opportunities for unlicensed users. Obviously, having to modify the MD-CAN operation to our needs means that it will no longer conform to standard MD-CAN implementation but rather a geometric multi-dimensional data representation with limited CAN functionality.

2) Historical Statistics Database: the MD-CAN-stored data is subject to constant changes, meaning users may come and go, hence changing communication relationships. Therefore, e2e path computations need to be based not solely on current available operational parameters, but also on statistics computed over longer time periods for a group of users. Having the Historical Statistics Database maintaining this data enables the Decision Maker algorithms to learn from experience and predict future changes more accurately.

3) Decision maker: this is used for end-to-end (e2e) route computation. Given the information provided from the MD-CAN and Historical-Statistics DB overlays, an e2e source-based route can be computed according to user preferences. The e2e path is computed according to decisions made with reference to a particular temporal framing and various parameters, e.g., QoS/QoE, cost, service availability, security and privacy levels. Different mechanisms are used in decision making, e.g., contextaware, fuzzy logic, analytic hierarchy processing [10]. The compiled e2e path is propagated back to the CRD.

4) CRD-Communication: all communication between the $\mathrm{SN}$ itself and the terminal (CRD) is handled by this overlay. The communication is done over a Common Control Channel (CCC) and through specific response types conceptualized to handle all necessary data exchange, e.g., bootstrap response, adaptation response, update response and CRD communicate response. This overlay is thus responsible for collecting all required information from the necessary overlays and properly format the CRD response messages before they are sent out on the CCC.

5) Additional functionality provided by the Support Node is, e.g., bootstrapping procedures for joining unlicensed CR users (including a bootstrap scheduler in order to fairly choose among the CRDs wishing to join the CRN), keeping the MD-CAN topology consistent through the 
updates received from the CRN members, keeping a member list of all current network members and handling inter-domain communication to other support nodes. The inter-domain communication to other SNs is either done via the SDR (communicating over the available frequency spectrum with SNs located nearby), or via the Internet communicating with SNs located far away.

\section{E. Common Control Channel}

Taking into consideration the dynamic properties of CRNs an out-of-band frequency in the unlicensed spectrum, the socalled Common-Control Channel (CCC), can be used covering long distances though operating at low rates [1], [7]. Replacing the need for large-scale broadcasts over multiple channels, the CCC must be available to all nodes in the network at all times. It is devised to minimize the overhead and the need for unnecessary flooding of the network while communicating with the SN. Furthermore, carrying all control information on a single channel eliminates synchronization problems, which otherwise can arise from having users tuned to different channels. To minimize the risk of interference we suggest to place the CCC in the unused parts of the UHF band [11]

\section{Multi-Dimensional CAN}

The suggested architecture demands that the data for e2e path computation and adaptation for CRN members is stored at the SN. This data is represented with the help of a MDCAN and updated by the CR users via the CCC. Member nodes only have information about their immediate neighbors. Furthermore, only affected CRDs send update information to the SN, keeping so the MD-CAN topology updated. The drawback of this architecture is that a centralized SN storing global network information indicates a single point of failure. This problem can later be solved by introducing a number of rendez-vous points (RP) to take over limited operations of the primary SN in case it fails. The RPs are well-connected nodes with static holding times with variations in the order of days and can hence handle bootstrapping and lookup procedures if necessary. Naturally, in case of primary SN failure the centralized management of available resources and optimized routing path computations are rendered invalid as well.

As mentioned, part of the CRN communication solution is based on an modified MD-CAN architecture responsible for addressing and information representation of a multihop CR-network. In regular CAN implementation, member nodes have $\Theta(2 d)$ neighbors and the average path lengths are of $\Theta(d / 4)\left(n^{1 / d}\right)$ hops [6]. CAN is a robust, scalable and decentralized Internet-scale hash table designed for efficiently locating data stored in a DHT. In general, by adding a multidimensional extension to the standard CAN implementation we gain the benefit of shorter path lengths at the price of increased number of per-node-states [6]. Every node keeps track of its neighbors in all neighboring dimensions.

By considering a regular MD-CAN to use for our particular needs, we suggest a flexible solution for the management of
CRNs. Different CAN dimensions can be used to represent different CR-dimensions like, e.g., space, frequency, power, code. A vacant position (zone) in a CAN dimension represents a hole in the particular CR-dimension as a function of time. No regular CAN takeover mechanisms are invoked for taking over the free zone [6]. This is simply an available resource that is handed over to the next CR user waiting to join the CRN and receive service. Naturally, by changing parts of a standard MD-CAN to our needs, means that it may no longer conform to regular MD-CAN operation. Instead it can be viewed as a geometric multi-dimensional data representation and addressing system with limited CAN functionality.

The main operations are:

- To impose a specific network structure over a geographical radius and represent its attributes (including free holes) as CAN zones.

- To represent operational parameters per CRD, e.g., data representation.

- To compute a multi-constrained multi-objective e2e path in collaboration with the Historical Statistics DB for communicating CRDs.

Stored operational parameters for a node in different CR-dimensions are identified with the Cartesian coordinates $n(t)=(x(t), y(t), f(t), p(t))$, where $x(t)$ and $y(t)$ denote the space dimension (we assume a 2-dimensional space, though a third space dimension might be required for depicting height), $f(t)$ the frequency dimension (partitioned into channels) and $p(t)$ the power dimension. All these parameters are functions of time. This means that $n(t)$ can, at any given time, characterize a CRD occupying the operational zone at coordinates $x, y$ in the space dimension, channel $c$ in the frequency dimension and using transmission output power $p$ in the power dimension. The defining characteristics of each dimension can also be changed independently of each other. Optimization can hence be achieved from the perspective of every single CR user, enabling pro-active adaptation to network changes.

Furthermore, users might need to adapt in one or more CRdimensions to keep the location in the space dimension. For instance, a slight change of the operating frequency or the transmit power output might be required to maintain a position in the space dimension and thus retain service. An example scenario of CRD adaptations can be as following: to retain the operational zone $<x_{2}, y_{1}>$ in the space dimension an adaptation in the frequency and power dimensions may be necessary, for instance from: $<x_{2}, y_{1}, f_{4}, p_{5}>$ to $<x_{2}, y_{1}, f_{9}, p_{3}>$. These adaptations are bounded by $t$ and represented as MDCAN dimensions. In other words, this is spectrum mobility represented as a multi-dimensional adaptation process with the goal of providing smooth and fast transition, with a minimum of e2e performance degradation [2].

In our approach, the frequency domain is represented as a CAN dimension named channels. This allows us to depict all available operation opportunities (channels) in the available frequency spectrum. Depending on the employed technology, different waveforms are used in the available frequencies enabling CR users to exploit the same frequency though at 
different time slots. The channels dimension sets an upper limit to the amount of users that can simultaneously occupy the available spectrum.

Given the spectrum $S \supset\left\{\Delta f_{1}, \Delta f_{2}, \Delta f_{3}, \ldots \ldots . \Delta f_{n}\right\}$, where $\Delta f_{1}$ to $\Delta f_{n}$ are available frequency ranges (in $\mathrm{Hz}$ ) within the available spectrum $S$ in a particular SN coverage area, we have the total number $C_{t o t}$ of available channels in a current SN coverage area:

$$
C_{t o t}=c\left(\frac{1}{M_{k n}} \sum_{i=1}^{n} \Delta f_{i}\right)
$$

where $\Delta f_{i}$ indicates available frequency range $i$ within available spectrum $S$ at current location. $M_{k n}$ represents the minimum amount of frequency bandwidth in $\mathrm{Hz}$ (including guard band) required for setting up a communication channel between two arbitrary CR users $k$ and $n$. Parameter $c$ denotes the maximum number of channels that can be set up within the same frequency range depending on the used waveform.

Moreover, since the frequency spectrum is a finite resource, an upper limit to the amount of simultaneously serviced CRDs can be reached. In order for the MD-CAN optimization approach to work, a bounded number of occupants is required per dimension, i.e., every user has to be represented in every CANdimension. Consequently, the frequency dimension limit is also the upper limit for the amount of simultaneous occupants in the remaining CAN-dimensions. Naturally, even though the number of users is bounded in every dimension, it is still possible for users present in the power dimension to occupy the same power zone. Meaning, more than one user might need to transmit at the same power output level for the moment, thus occupying the same power zone but being in different, e.g., frequency zones (occupying different channels).

\section{Cognitive Engine}

The cognitive engine is defined to represent a set of algorithms that together perform sensing, learning, optimization and adaptation control of the CRN [12]. In other words, the cognitive engine is an intelligent wireless communication system able to continuously:

- Perceive the radio environment in continuous time

- Learn from the radio environment

- Facilitate the communication among multiple users

- Adapt the e2e performance to statistical variations of the radio environment as well as user preferences and behavior

- Facilitate self-adapting manner for communication

- Solve diverse conflicts among users

- Provide self-awareness

In our case, several operations are performed in sequence by a set of algorithms at every CRD and SN, as shown in figure 2 . These operations are outlined in Algorithms 1, 2 and 3.

Algorithms 1 and 2 show the procedures implemented at CRDs whereas Algorithm 3 the procedures implemented at SNs. A brief description of the functionality is presented

\begin{tabular}{l|l|l|l|l|}
$\mathrm{Ca}$ & $\mathrm{Sa}$ & $\mathrm{A}$ & Icrd & Dcrd \\
\hline
\end{tabular}

TABLE I

COMMON FORMAT CRD MESSAGE TYPES.

at the begining of every procedure. Procedure names preceded by a variable name and a dot indicate remote function calls, e.g., Csn.SNMAIN $(C a, S a, A, C c r d, D c r d)$ means that the current CRD with UUID $C c r d$ executes the function SNMAIN $(C a, S a, A, C c r d, D c r d)$ on the current SN with UUID Csn.

Table I shows the common format (CF) for the used CRD message types bootstrap message adaptation message, update message and CRD communicate message used (Algorithm 1 and Algorithm 2). $C a$ is control data, $S a$ is sensing data, $0 \leq A \leq 3$ is CRD message type information, Icrd is the ID of the source CRD and Dcrd is the ID of a destination.

These parameters refer to the following:

- Bootstrap $(A=2)$ : the $C a$ and $S a$ parameters contain the particular CRDs sensing and control capabilities. The value of variable $A$ informs the SN that this is a bootstrapping request from a CRD with ID I $c r d$. The Derd value is undefined.

- Adaptation $(A=1)$ : the $C a$ and $S a$ parameters contain newly collected control and sensing data. The value of variable $A$ informs the $\mathrm{SN}$ that this is an adaptation request from a CRD with ID Icrd. The Dcrd value is undefined.

- Update $(A=0)$ : the $C a$ and $S a$ parameters contain newly collected control and sensing data. The value of variable $A$ informs the $\mathrm{SN}$ that this is an operational parameters update message from CRD with ID I crd. The Dcrd value is undefined.

- Communicate $(A=3)$ : in this case the $S a$ parameter is undefined whereas the $C a$ parameter contains the minimum bandwidth required for the particular service Bmin, the upper bound of the minimum required delay D $\max$ and the maximum cost allowed $C \max$ for the particular service. The value of variable $A$ informs the $\mathrm{SN}$ that this is a request from CRD with ID Icrd to communicate with CRD with ID Dcrd, i.e., send data there.

All CRDs are assumed to implement the procedures GETSEnSingParameters() and GetControlParameters(). These procedures collect all necessary control and sensing parameters, returning them as two separate arrays. Procedure ISADAPTATIONNEEDED $(S a, C a)$ uses data stored in the returned arrays to determine if adaptations to a CRDs operational parameters are needed, returning true(1) or false(0).

Given the complexity of the complete routing algorithm, only the basic operations are presented here. Finally, the procedure COMMUNICATE() checks if a CRD wishes to communicate to another $\mathrm{CRD}$ in the $\mathrm{CRN}$, returning either 1 or 0 .

The Support Node is responsible for handling many important operations, i.e., providing bootstrapping procedures for 


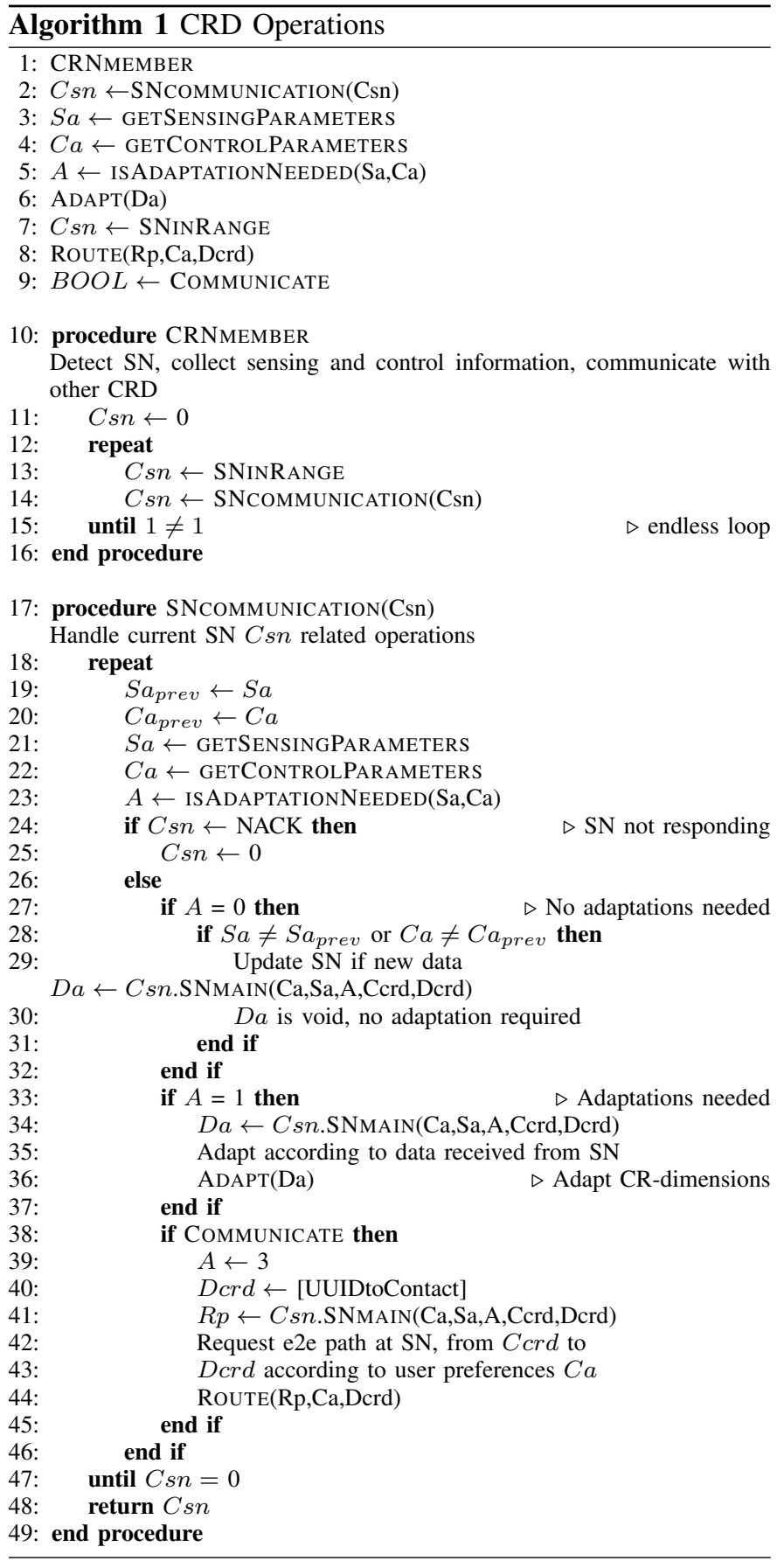

arriving unlicensed CR users, keeping the MD-CAN structure consistent and valid through the updates received from the CRN members, compile statistics for a group of CR users, build up a Historical Statistics Database over a period of time, compute e2e routes and handle inter-domain communication to other support nodes handling other CR user batches. These operations are outlined in Algorithm 3.

Table II shows the $\mathrm{SN}$ response message types used in Algorithm 3: update, adaptation, bootstrap, communication. All SN response messages are returned as data-structure $D a$. The included data is adapted depending on the particular

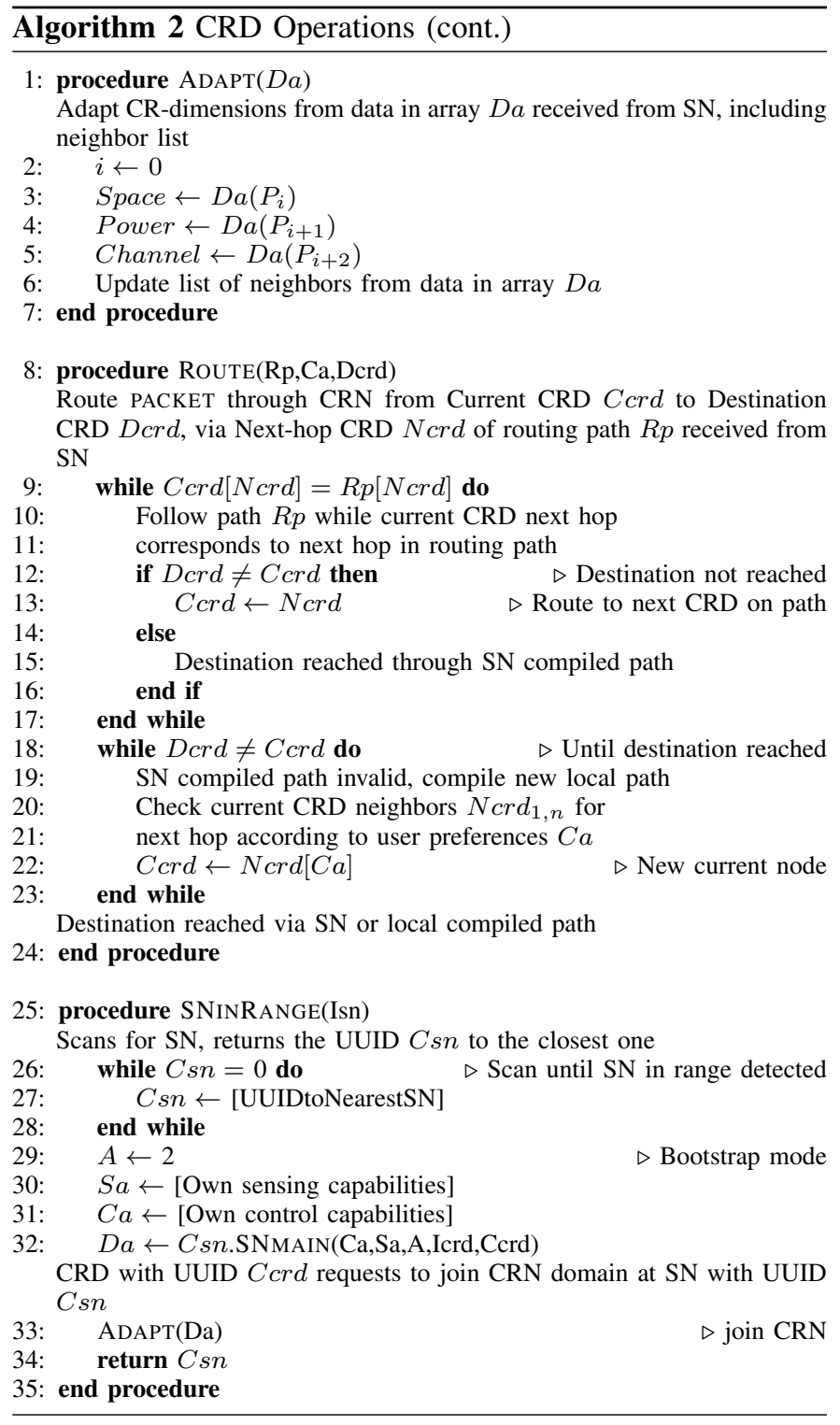

response type.

- Update: the Ccrd is the ID of the CRD that the response is aimed for.

- Adaptation and Bootstrap: adaptation and bootstrap responses are identical. The TxPower contains new transmit output power levels, whereas Channels comprises the channel assignment for the requesting CRD (including MAC and physical layer data) [9]. CAN coord contains the cartesian coordinates of the newly assigned geographical zone. The NeighborList is the updated neighbor table of the requesting CRD. The Ccrd is the ID to the CRD that the response is aimed for.

- Communicate: the IntermediateNodes includes all intermediate nodes (CRDs) along the path to the destination.

TxPowerPerHop and ChannelsPerHop contain the adaptations needed to the transmit power output and 


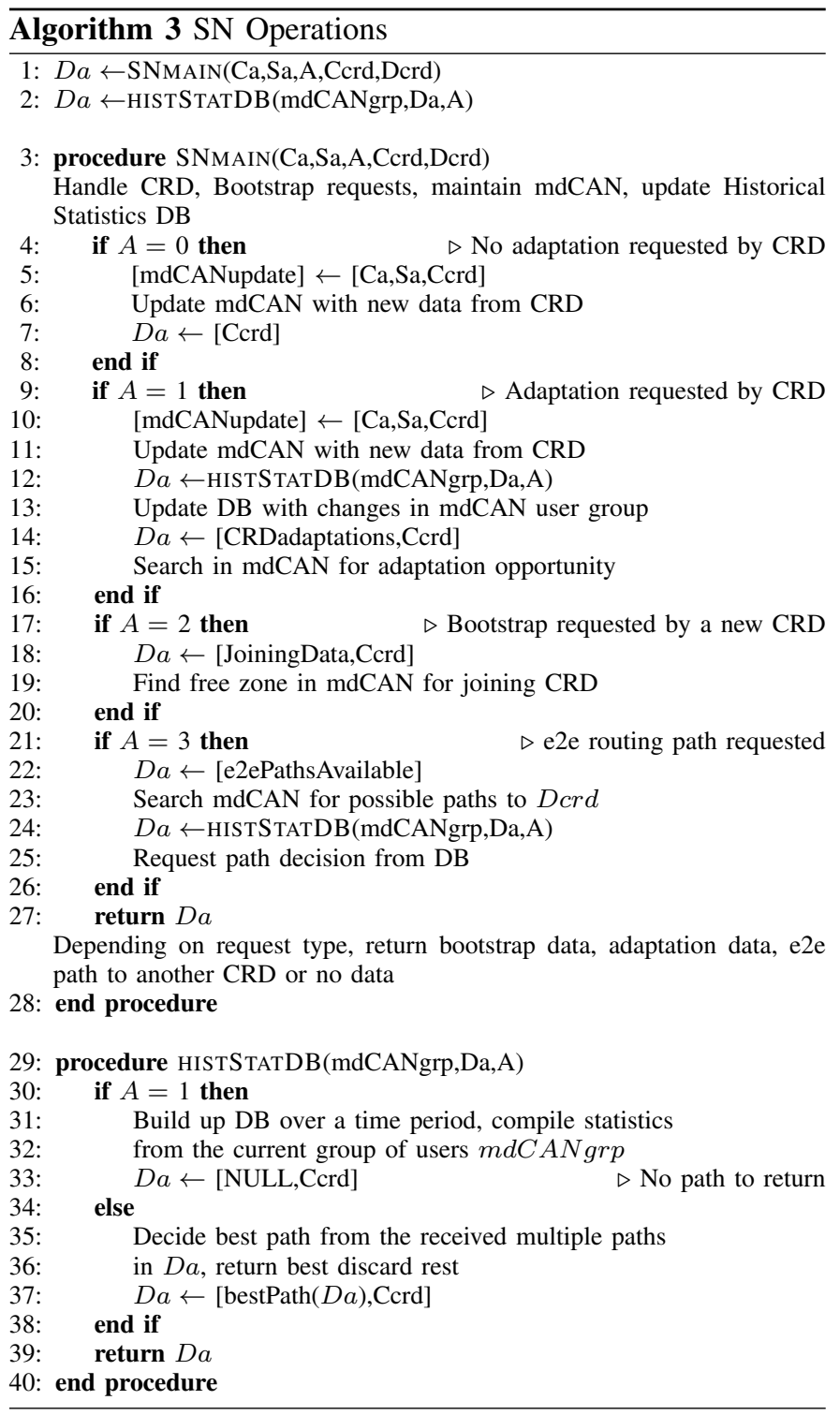

channel assignment (including MAC and physical layer data) for all nodes along the e2e path. These adaptations are compiled according to user preferences and environmental constraints. Dcrd is the ID of the destination CRD and $C$ crd is the ID of the source CRD.

The algorithm for selecting the best path $i$ from $n$ available paths (line 37, Algorithm 3) can be expressed as a weighted utility function $F\left(p_{i}\right)$ of three average global metrics (bandwidth, delay and cost) [13]. The metrics are first computed for each available e2e path between a particular source and destination. The obtained values for every path are used as references to compare against user preferences. In other words, the user determined requirements of bandwidth, delay and cost (expressed as averages) for a particular e2e path are weighted against the computed average values of the available paths between the specific source and destination nodes. The path $p_{i}$ with highest degree of correlation to the user determined preferences is selected and the rest are pruned. We define the

\begin{tabular}{|l|l|l|}
\hline Update & Da & Ccrd \\
\hline Adaptation & Da & $\begin{array}{l}\text { TxPower } \\
\text { Channels } \\
\text { CANcoord } \\
\text { NeighborList } \\
\text { Ccrd }\end{array}$ \\
\hline Bootstrap & Da & $\begin{array}{l}\text { TxPower } \\
\text { Channels } \\
\text { CANcoord } \\
\text { NeighborList } \\
\text { Ccrd }\end{array}$ \\
\hline Communicate & Da & $\begin{array}{l}\text { IntermediateNodes } \\
\text { TxPowerPerHop } \\
\text { ChannelPerHop } \\
\text { Dcrd } \\
\text { Ccrd }\end{array}$ \\
\hline
\end{tabular}

TABLE II

SN RESPONSE MESSAGE TYPES

path utility function as being:

$$
\begin{aligned}
F\left(p_{i}\right) & =\gamma_{B} \max \left[\left(1-\frac{B p_{i}}{B u}\right), 0\right] \\
& +\gamma_{D} \max \left[\left(\frac{D p_{i}}{D u}-1\right), 0\right] \\
& +\gamma_{C} \max \left[\left(\frac{C p_{i}}{C u}-1\right), 0\right]
\end{aligned}
$$

where parameter $B u$ denotes the minimum average bandwidth required for a service (path) by the user, and $B p_{i}$ is the average bandwidth for path $i$. Parameter $D u$ is the user determined upper bound of the accepted average delay for a service (path), and $D p_{i}$ is the average delay for path $i$. Parameter $C u$ represents the maximum average cost set by the user that is accepted for a service (path), and $C p_{i}$ is the average cost for path $i$.

The suitability of a path is seen from the deviation between the user defined average preferences (for bandwidth $B u$, delay $D u$ and cost $C u$ ) and the corresponding averages for the available paths $\left(B p_{i}, D p_{i}\right.$ and $\left.C p_{i}\right)$. The $F\left(p_{i}\right)$ utility function expresses the suitability for path $i$ by weighting deviations of the considered metrics through the constant parameters $\gamma_{B}$, $\gamma_{D}$ and $\gamma_{C}$ (for bandwidth, delay and cost, respectively) [13], [14]. Furthermore, for bandwidth, the large averages (of the available paths) are normalized to zero whereas for delay and cost the small averages are normalized to zero. In other words the more suitable an available path is (according to user preferences), the closer to zero the utility function $F\left(p_{i}\right)$ gets (meaning the less the deviations to user request are).

\section{Routing in Cognitive Radio Networks}

Considering the large diversity of network configurations and network conditions together with user activities it is clear that routing in cognitive radio networks is very challenging [7]. Variations in the available frequency bands may lead to large variations in the topology and connectivity map of the CRN. Hence, finding an appropriate path between a particular source node and a particular destination node becomes extremely 
complicated. We can partition the $\mathrm{CRN}$ routing in three categories by considering the activity and holding times of licensed users as a reference framing [1], [2], [7]:

- Static routing, used given that the holding times of the licensed bands are relatively static processes, i.e., with variations in the order of hours or days. Although this shows resemblance to multi-radio multi-channel mesh networks, there is a need to deal with transmissions over parallel channels as well as handling interference among licensed users and unlicensed users.

- Dynamic routing, used given that licensed bands are intermittently available. Smaller temporal framings, in the order of hours down to minutes, demand for more dynamic routing decisions. The main challenges are to find stable routing paths able to provide the expected e2e performance and to define appropriate routing metrics able to capture spectrum fluctuations. The used routing algorithms are in this case similar to those for wireless ad-hoc networks, i.e., combinations of static mesh routing and per-packet dynamic routing.

- Opportunistic (or highly dynamic) routing, used because the licensed bands show very high variations with temporal framings in the order of minutes down to seconds. This demands for routing algorithms able to take quick, local decisions. Solutions encompass per-packet dynamic routing over opportunistically available channels. The routing is adapted in this case to the particular conditions that exist at a specific time moment. This type of routing is also called self-aware routing [15].

A combined routing solution is suggested for $\mathrm{CRN}$ routing. The Decision Maker overlay at the SN uses a static routing model to locally compile the e2e routing path. By having every $\mathrm{CRD}$ connecting to the $\mathrm{SN}$ and receiving an e2e path compilation before starting a communication, unnecessary flooding can be avoided and accurate source-based routing paths can be computed. This is possible since the information stored locally in the MD-CAN and Historical Statistics Database at the $\mathrm{SN}$ is continuously updated (through the CCC from all CRN members) with all vital data to reach requested destinations, including channel assignment.

The computed path takes into account the available resources, user preferences, environmental constraints and even predictions of future network adaptations. As future work we plan to develop a prediction overlay that can learn from previous experience and thus perform predictions with increased accuracy over time. This further improves the e2e path compilations leading to compiled routes that hold for the expected time period.

After receiving the e2e routing path from the $\mathrm{SN}$, a CRD forwards a packet to neighbors along the path (through the information written in the packet header). Due to the approach used for an e2e source-based route compilation in a multi-hop scenario, there is a risk that a destination becomes unavailable before it is reached. Packets are then either discarded according to a Time-to-Live (TTL) counter added to all packets sent on the network or by an intermediate node having more upto-date knowledge of the destination availability.

However, if the precompiled e2e path becomes invalid while on route due to the mobility of $\mathrm{CR}$ users (arriving licensed users might push out unlicensed users that are part of the e2e path, rendering it invalid), local routing decisions have to be made, adapting the precompiled e2e path according to the particular network conditions and user preferences, e.g., cost or throughput.

Finally, a way to prioritize between unlicensed users competing for free spaces needs to be devised. Such a solution can be based on cost (users who can afford the available spectrum hole to receive service), power (users who can meet the required power outputs for the available spectrum hole to receive service), security level or a balanced approach (several unlicensed users can share the cost and the available spectrum).

\section{Multi-Dimensional Routing And Optimization}

There are three essential parts related to the problem of routing in CRNs. These are the definition of an appropriate QoS metric for the e2e routing, the path selection algorithm and the routing algorithm. A particular difficulty in our case is because an e2e route may involve crossing different spaces, e.g., geographical space, frequency and power. In general, the path selection problem is viewed as an optimization problem. A network is in this case represented by a directed graph $G=$ $(N, E)$ where $N$ is a set of vertices (nodes) and $E$ is a set of directed links (edges).

Each link is associated with a set of additive QoS metrics (e.g., delay, cost) and non-additive QoS metrics (e.g., bandwidth). We assume the number of QoS metrics as being denoted by $m$. Each link is therefore characterized by a $m$ dimensional link weight vector, which consists of $m$ nonnegative QoS weights as components. Accordingly, the QoS measure of an e2e path can be either additive (e.g., delay) or minimum of the QoS weights along the particular e2e path (e.g., bandwidth). Problems involving constraints on nonadditive metrics can easily be solved by pruning the links of the graph not satisfying the particular constraint. On the other hand, additive QoS metrics are more difficult to handle, and demands for solving sophisticated optimization problems, e.g., Multi-Constrained Paths (MCP) optimization problem and Multi-Constrained Optimal Path (MCOP) optimization problem [16], [17].

Since the optimal solutions for such type of problems is NP-complete, heuristics or approximation algorithms will be used. Several popular algorithms considered in our research are Self-Adaptive Multiple Constraints Routing Algorithms (SAMCRA) [17] and Particle Swarm Optimization [18]. Given the suggested CRN architecture, our focus is on path selection algorithms that can handle multiple constraints (with particular focus on linear constraints) and multiple objectives (e.g., simultaneous minimal delay and maximum bandwidth). Furthermore, we are also focusing on the design of multi-dimensional routing algorithms, able to provide e2e routes along several 
dimensions, e.g., geographical space, frequency and power. Furthermore, the e2e routing algorithms should cope with dynamic environments, e.g., resource fluctuations, churn. Thus these algorithms have hard "realtime" performance demands, meaning they are requested to compute new feasible routes for each link-state update.

Two possibilities for the e2e routing are available, namely either by serializing routing in multi-dimensional space based on a composed QoS metric or by doing routing in onedimensional space combined with mapping from the multidimensional space into the one-dimensional space together with the mapping of the associated QoS metrics [19]-[21]. These routing alternatives will be investigate and compare together with the associated optimization algorithms. We will furthermore use different traffic models and network conditions in these experiments, to help us in designing routing and optimization algorithms able to cope with realistic traffic and networking conditions [22].

\section{CONCLUSIONS}

A novel architectural solution has been advanced for the efficient management of CRNs. The architecture is based on the use of a middleware with a common set of APIs, a number of overlay entities and a multi-dimensional routing. The architecture is described with emphasis on the associated research challenges, such as sensing and prediction, addressing and routing, middleware and decision making. More specifically, two of the most important elements of this architecture are the solutions adopted for MD-CAN and the CRN routing focusing on the local modeling of CRN routing in case of e2e path failure.

Future work is two-fold. Firstly, analytical and simulation models will be devised towards the performance evaluation and validation of the suggested architecture with a particular focus on the routing mechanisms. Secondly, this architecture will be implemented and tested using appropriate platforms, under different traffic conditions [22].

\section{ACKNOWLEDGMENTS}

The authors gratefully acknowledge the support of Euro-NF Network of Excellence, for the work presented in this paper. The work was done as part of the Euro-NF project Networking over Cognitive Radio Networks (NETCO).

\section{REFERENCES}

[1] I.A. Akyildiz, L. Won-Yeol, and K. Chowdhury, "Spectrum managemen in cognitive radio ad hoc networks", IEEE Network, vol. 23, no. 4, pp. 6-12, 2009.

[2] I.A. Akyildiz, L. Won-Yeol, and K. Chowdhury, "Crahns: Cognitive radio ad hoc networks", Ad Hoc Networks, vol. 7, no. 5, pp. 810-836, 2009.

[3] T. Yucek and H. Arslan, "A survey of spectrum sensing algorithms for cognitive radio applications", IEEE Communications Surveys \& Tutorials, vol. 11, no. 1, pp. 116-130, 2009.

[4] R.W. Thomas, L.A. DaSilva, and A.B. MacKenzie, "Cognitive networks", in First IEEE International Symposium on New Frontiers in Dynamic Spectrum Access Networks, 2005. DySPAN 2005, 2005.

[5] L. Khalid and A. Anpalagan, "Emerging cognitive radio technology: Principles, challenges and opportunities", Computers \& Electrical Engineering, vol. 36, no. 2, pp. 358-366, 2010.
[6] S. Ratnasamy, P. Francis, M. Handley, R. Karp, and S. Shenker, "A scalable content-addressable network", in SIGCOMM '01: Proceedings of the 2001 conference on Applications, technologies, architectures, and protocols for computer communications, New York, NY, USA, 2001, pp. 161-172, ACM.

[7] H. Khalifé, N. Malouch, and S. Fdida, "Multihop cognitive radio networks: to route or not to route", IEEE Network: The Magazine Of Global Internetworking, vol. 23, no. 4, pp. 20-25, 2009.

[8] A. P. Popescu, D. Erman, K. de Vogeleer, and A. O. Popescu, "Roma: A middleware framework for seamless handover", in Proceedings of 2nd EuroNF Workshop and Future Internet Cluster meeting, 2009.

[9] B. Ackland, D. Raychaudhuri, M. Bushnell, C. Rose, I. Seskar, T. Sizer, D. Samardzija, J. Pastalan, A. Siegel, J. Laskar, S. Pinel, and K. Lim, "High performance cognitive radio platform with integrated physical and network layer capabilities", Tech. Rep. NSF grant CNS-0435370, WINLAB, Rutgers University, July 2005.

[10] M. Kassar, B. Kervella, and G. Pujolle, "An overview of vertical handover decision strategies in heterogeneous wireless networks", Computer Communications, vol. 31, no. 10, pp. 2607-2620, 2008.

[11] P. Bahl, R. Chandra, T. Moscibroda, R. Murty, and M. Welsh, "White space networking with wi-fi like connectivity", ACM SIGCOMM Computer Communication Review, vol. 39, no. 4, pp. 27-38, 2009

[12] B. Le, W. T. Rondeau, and W. C. Bostian, "Cognitive radio realities", Wireless Communications and Mobile Computing, vol. 7, no. 9, pp. 1037-1048, 2007.

[13] T. Ciszkowski, W. Mazurczyk, Z. Kotulski, T. Hossfeld, M. Fiedler, and D. Collange, "Towards quality of experience-based reputation models for future web service provisioning", in Future Internet Architectures: New Trends in Service Architectures (2nd Euro-NF Workshop), Santander, Spain, 62009.

[14] K. Tutschku, M. Fiedler, S. Chevul, O. Radtke, and A. Binzenhoefer, "The network utility function: A practicable concept for assessing network impact on distributed services", Tech. Rep., University of Wrzburg, 2005.

[15] Erol Gelenbe, "Steps toward self-aware networks", Communications of the ACM, vol. 52, no. 7, pp. 66-75, 2009

[16] D. Ilie and A. Popescu, "A framework for overlay qos routing", 4th Euro-FGI Workshop on New Trends in Modelling, Quantitative Methods and Measurements, 2007, http://www.bth.se/fou/Forskinfo.nsf/ Sok/6d5f6f18243f6433c125730e006eaa15!OpenDocument

[17] P. Van Mieghem and F. A. Kuipers, "Concepts of exact qos routing algorithms", IEEE/ACM Transactions on Networking (TON), vol. 12, no. 5, pp. 851-864, 2004.

[18] A. P. Engelbrecht, Fundamentals of Computational Swarm Intelligence, John Wiley \& Sons, 2006.

[19] M. Cai, M. Frank, J. Chen, and P. Szekely, "Maan: A multi-attribute addressable network for grid information services", in Fourth International Workshop on Grid Computing, 2003, pp. 184-191.

[20] M. Subhash, N. Yuan, and S. Chong, "Range and knn searching in p2p", 2004, http://www.comp.nus.edu.sgl ooibc/courses/cs6203/ RangeandkNN.pdf.

[21] P. Ganesan, B. Yang, and H. Garcia-Molina, "One torus to rule them all: multi-dimensional queries in p2p systems", in 7th International Workshop on the Web and Databases, New York, NY, USA, 2004, pp. 19-24, ACM.

[22] D. D. Kouvatsos, Traffic and Performance Engineering for Heterogeneous Networks, River Publishers, 2009.

[23] S. Arkoulis, M. Fiedler, P.A. Frangoudis, R. Herkenhöner, G.F. Marias, H. de Meer, and G.C. Polyzos, "Distributed sensing for spectrum agility: Incentives and security considerations", in Proceedings of Euro-NF, 2008.

[24] J. N. O'Daniell, Analysis and design of cognitive radio networks and distributed radio resource management algorithms, $\mathrm{PhD}$ thesis, Blacksburg, VA, USA, 2006, Adviser-Reed, Jeffrey H.

[25] K. M. Powell, J. M., J. K. Martin, and S. J. Adelstein, "Notice of proposed rule making and order: Facilitating opportunities for flexible, efficient, and reliable spectrum use employing cognitive radio technologies", Federal Communications Commission, Notice Of Proposed Rule Making And Order, 2003, http://www.fcc.gov/.

[26] J. Mitola, III, Cognitive Radio: An Integrated Agent Architecture for Software Defined Radio, PhD thesis, Electrum 204 SE-164 40 Kista, Sweden, 2000. 